\title{
Long term growth arrest of human Tenon's fibroblasts following single applications of $\beta$ radiation
}

\author{
P H Constable, J G Crowston, N L Occleston, M F Cordeiro, P T Khaw
}

\begin{abstract}
Aims/background-Antimetabolites are increasingly used to manipulate the healing response after filtration surgery, but problems with thin cystic blebs have been encountered with the liquid agents commonly used such as 5-fluorouracil and mitomycin C. $\beta$ Radiation appears to be a useful adjuvant treatment for preventing scarring after trabeculectomy, resulting in diffuse rather than cystic bleb formation, but much of the basic cell biology of the ocular fibroblast response to $\beta$ radiation remains unclear. The effects of $\beta$ radiation on ocular fibroblast proliferation and cell cycling were investigated to determine the nature and duration of these effects on these cells.

Methods-In vitro cell culture techniques were used to investigate fibroblast proliferation. Cell viability was studied using trypan blue dye exclusion. The effect of radiation on cell cycling was investigated using bromodeoxyuridine uptake. p53 expression was demonstrated using immunocytochemistry .

Results- $\beta$ Radiation inhibited fibroblast proliferation in a dose dependent manner. Early cell death was not a prominent feature, but irradiated fibroblasts demonstrated a rapid onset and sustained period of growth arrest. p53 expression was found to be increased in irradiated cells.

Conclusions-Single doses of $\beta$ radiation significantly inhibit Tenon's capsule fibroblast proliferation in vitro over a 28 day period. This inhibition is the result of a rapid onset and sustained period of growth arrest in irradiated cells. Irradiated fibroblasts show an increase in p53 expression, a nuclear phosphoprotein which has been associated with control of the cell cycle. Single applications of $\beta$ radiation may be an effective treatment for the prevention of bleb failure as a result of prolonged growth arrest of Tenon's capsule fibroblasts.

(Br F Ophthalmol 1998;82:448-452)
\end{abstract}

Peroperative antimetabolite treatments have been used at the time of trabeculectomy to prevent early postoperative failure in high risk patients, ${ }^{1-3}$ and increasingly to help achieve optimal intraocular pressure (IOP) reductions in many other patients. The antimetabolites 5-fluorouracil and mitomycin C are most commonly used for these treatments, but as they are used in a liquid form their use may be complicated by intraocular or extraocular leakage, with potentially serious toxic side effects. In addition, the avascular cystic blebs often seen after antimetabolite use can lead to complications such as hypotonous maculopathy and infective endophthalmitis. ${ }^{4-7}$ These problems have prompted research into alternative treatments which may also be effective in modulating the subconjunctival healing response after filtration surgery.

Previous clinical experience has shown that many irradiated tissues heal poorly, suggesting that radiation may be used therapeutically for modulating postoperative healing. $\beta$ Radiation delivered from a solid state probe can be focally delivered to the bleb site immediately following surgery, with the rapid attenuation of the $\beta$ particles making it ideal for treatment of such superficial tissues. Focal applications of up to 2500 cGy $\beta$ radiation have been found to be relatively well tolerated by the eye, and have previously been shown to be effective in preventing pterygium recurrence following surgical excision. ${ }^{89}$ Furthermore, the focal nature of the treatment has led to suggestions that $\beta$ radiation may be useful in the treatment of macula degeneration and in prevention of post-angioplasty stenosis. The earliest reports of $\beta$ radiation as an adjuvant treatment in filtration surgery date from the 1940 s, with several authors suggesting that this was an effective adjuvant therapy. ${ }^{10-12}$ In 1991, Miller and Rice reported that peroperative treatment with 750 cGy $\beta$ radiation significantly enhanced bleb survival and improved IOP control following trabeculectomy in congenital glaucoma. ${ }^{13}$ Furthermore, clinical experience of this treatment has suggested that the blebs which form after irradiation are generally diffuse, rather than cystic and avascular, and in particular the long term safety experience following this treatment has been excellent.

Despite the clinical evidence of its efficacy, however, much of the underlying cell biology of the effects of single doses of $\beta$ radiation on human Tenon's fibroblasts (HTFs) remains unknown. The first published report was by Nevarez et al, who reported that over a 7 day period, single doses of $\beta$ radiation significantly inhibited monkey fibroblast proliferation relative to unirradiated control cells. ${ }^{14}$ Khaw et al subsequently described a similar effect in human ocular fibroblasts over a 14 day period. ${ }^{15}$ More recently, Di Leonardo et al have also reported that treatment with single doses of gamma radiation resulted in a prolonged 


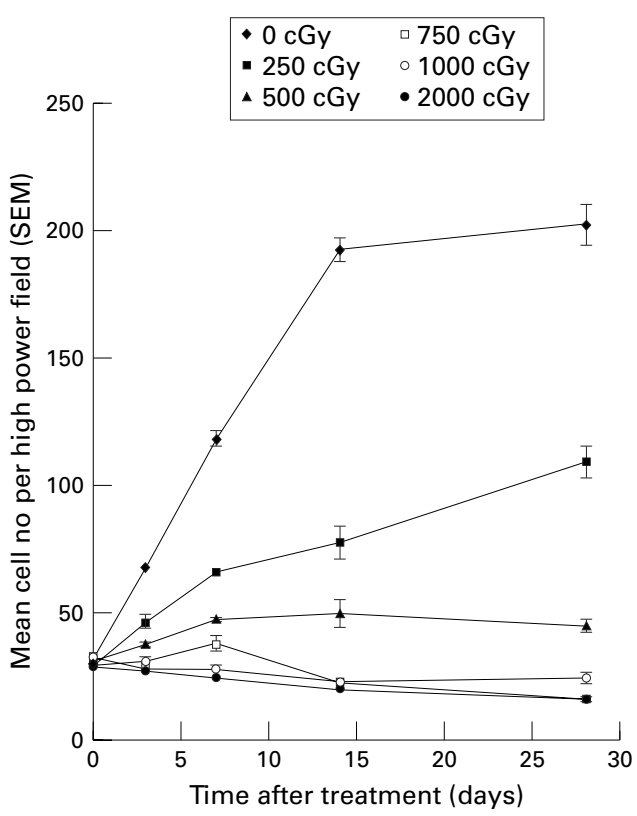

Figure 1 Effects of $\beta$ radiation on fibroblast proliferation. Fibroblasts were grown in tissue culture, passaged, and used for proliferation experiments when in exponential phase growth. The effect of single doses of $\beta$ radiation between 250 and 2000 cGy was investigated with control cells remaining unirradiated. At each time point, the mean number of human Tenon's fibroblasts (HTFs) in triplicate wells was determined for each dose of radiation studied. Single doses of $\beta$ radiation significantly inhibited HTF proliferation relative to control cells at all time points $(p<0.05$ at each time point using one way ANOVA with Bonferroni's modification).

period of growth arrest in several human fibroblast lines. ${ }^{16}$ In this study we have investigated the effects of $\beta$ radiation on HTF proliferation, viability, and cell cycling with a view to understanding the antiproliferative effects of $\beta$ radiation. We have also investigated the longer term effects of $\beta$ radiation on HTF proliferation, to establish if there is any late recovery of proliferation in the irradiated cells. In addition, we report on the effect of radiation on the expression of $\mathrm{p} 53$, a nuclear phosphoprotein which has been linked to cell cycle controls following DNA injury. ${ }^{17} 18$

\section{Materials and methods}

CELL CULTURES

Fibroblasts were cultured from donor explants in Dulbecco's modified Eagle's medium (DMEM) containing 10\% newborn calf serum (NCS, Gibco Life Technologies, UK) supplemented with L-glutamine ( $2 \mathrm{mM}$, Gibco, UK), amphotericin $(0.25 \mathrm{mg} / \mathrm{ml})$, gentamicin (50 $\mathrm{mg} / \mathrm{ml})$, and penicillin/streptomycin $(100 \mu \mathrm{g} /$ $\mathrm{ml})$, according to an established protocol. ${ }^{19}$ HTFs were grown to subconfluence, trypsinised, and passaged at a 1:3 ratio. Cells were used when in exponential phase growth for the proliferation assays, and used between the third and sixth passage.

CELL PROLIFERATION

A total of 2000 HTFs in DMEM supplemented with $10 \%$ fetal calf serum (FCS, Gibco Life Technologies), buffered with HEPES (Sigma UK), were cast into individual wells of

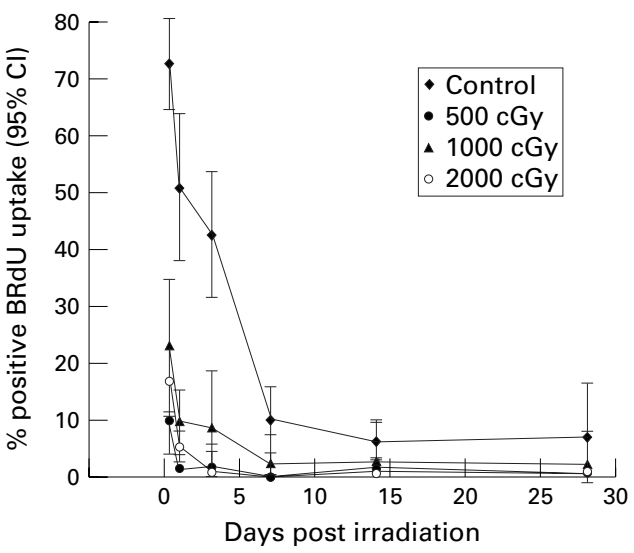

Figure 2 Cell cycling following $\beta$ irradiation. Fibroblasts were cast into 96 well plates and allowed to settle overnight. Test wells were irradiated with 500, 1000, or 2000 cGy, and control cells remained unirradiated. At various time points following treatment, triplicate wells from each treatment group were pulsed in 1:1000 BRdU in DMEM/10\% FCS for 24 hours, following which the incorporated BRdU was demonstrated by immunochemical antibody staining and $D A B$ precipitation. The mean percentage number of labelled cells in each treatment group, together with the $95 \%$ confidence interval, was plotted at the various time points. Statistical analysis performed using a one way ANOVA test with Bonferroni's modification demonstrated that fibroblast proliferation was significantly greater in the control group than in all irradiated populations at all time points $(p<0.05)$.

96 well plates and allowed to settle overnight. Wells to be treated were then placed in direct contact with the strontium-90 source (Amersham Life Sciences, UK), and various doses of $\beta$ radiation delivered to the cell monolayer. The dose of radiation was calibrated from the surface dose rate certificate issued by the manufacturer, and corrected for the thickness of the plastic in the plates using an ionisation chamber. Untreated control wells were sham treated, with each plate remaining out of the incubator for the same period of time. Single doses of 0, 250, 500, 750, 1000, and 2000 cGy $\beta$ radiation were delivered to a minimum of triplicate wells for each time point to be studied. The 96 well plates were then refed with fresh DMEM/10\% FCS and returned to the incubator. At each time point to be studied (day $0,3,7,14$, and 28) test wells were washed gently with phosphate buffered saline (PBS), fixed with methanol, and stained with a Giemsa stain. The number of fibroblasts in five randomly selected high power fields from each of the triplicate wells was counted, and the mean cell number per high power field and the standard error calculated for each treatment group at each time point.

Cell viability in each group was demonstrated by in situ vital dye exclusion. At each time point, triplicate wells for each dose studied were washed gently in PBS, and $0.4 \%$ trypan blue added for 5 minutes. The trypan blue was then washed off gently with PBS, and five randomly selected high power fields counted for viable and non-viable cells. Each experiment was repeated a minimum of three times.

BROMODEOXYURIDINE UPTAKE

HTFs were prepared and treated as above. At each time point, triplicate wells from each 

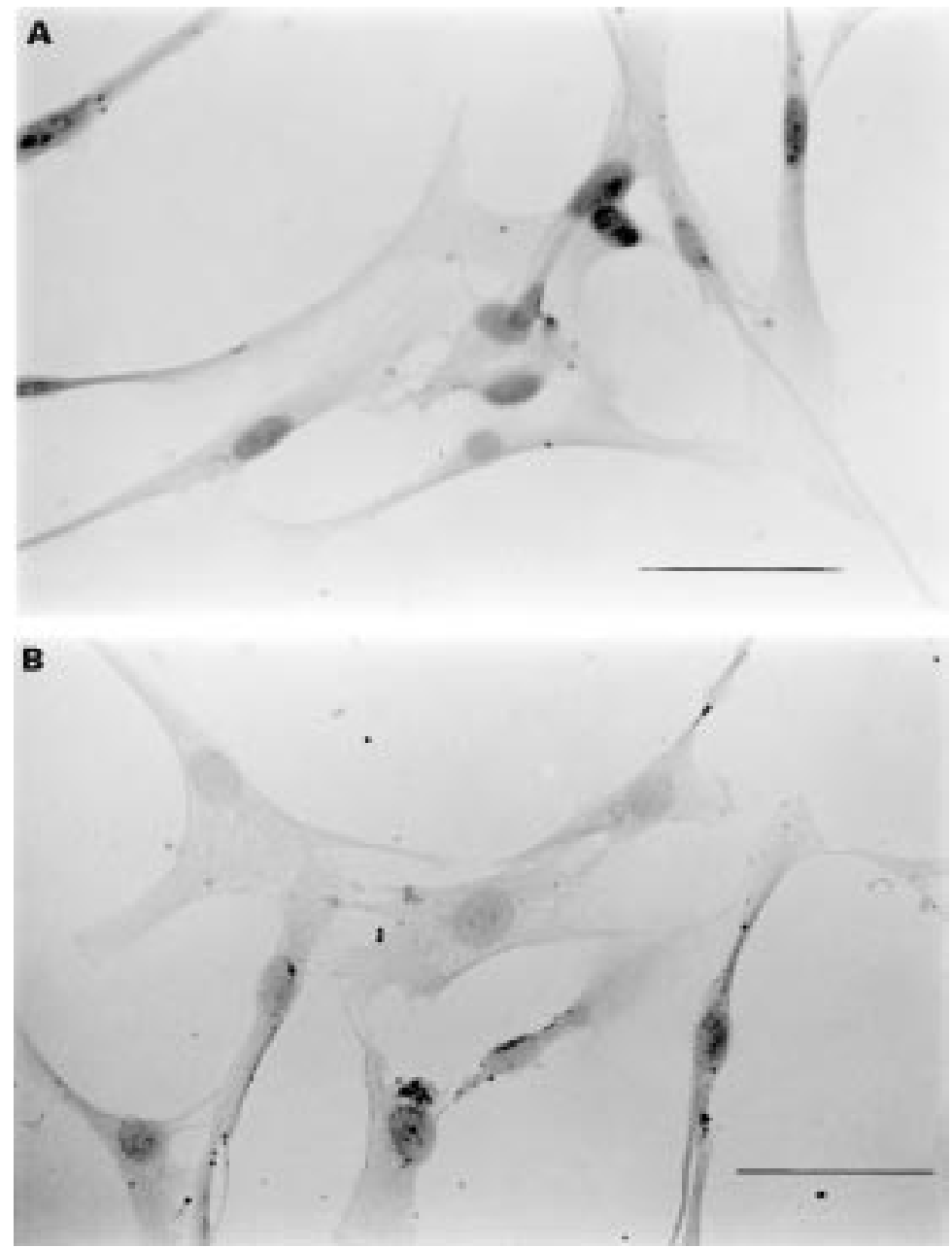

Figure 3 The effect of $\beta$ radiation on $p 53$ expression. (A) Irradiated fibroblasts at 24 hours (1000 cGy). Dark staining nuclei indicate positive p53 staining. (B) Unirradiated control fibroblasts at 24 hours. Bars $=50 \mu \mathrm{m}$.

treatment group were pulsed with $10 \mathrm{mM}$ bromodeoxyuridine (BRdU, Amersham UK) in DMEM $/ 10 \%$ FCS for 24 hours. The monolayers in the wells were then washed gently in PBS, fixed in glacial acetic acid/ethanol, and the incorporated BRdU demonstrated by immunocytochemistry using a monoclonal mouse anti BRdU antibody (Amersham UK) and immunoprecipitation with diaminobenzamine (DAB) giving a brown nuclear stain in positive cells. The cell monolayer was then counterstained with Meyer's haematoxylin, and the cycling index per well determined (number of positive cells per high power field/ total number of cells per high power field)..$^{20}$ HTF were studied 4 hours after irradiation, and at $1,3,7,14$, and 28 days after irradiation.

P53 STAINING

Monolayers of HTFs in 96 well plates were prepared as above and treated with single doses of $0,500,1000$, or 2000 cGy $\beta$ radiation. At 0 , $1,3,7,14$, and 28 days triplicate wells from each dose were washed with PBS, and then fixed with 50:50 acetone:ethanol for $10 \mathrm{~min}$ utes. The wells were then air dried and stored at $-20^{\circ} \mathrm{C}$ till needed. Wells were rehydrated with PBS for 10 minutes, and non-specific binding blocked with $3 \%$ bovine serum albu- min for 30 minutes. p53 staining was demonstrated using 1:100 monoclonal mouse antip53 antibody (Dako DO-7) for 12 hours, followed by 1:4000 peroxidase conjugated rabbit anti-mouse antibody (Sigma, UK) as the secondary antibody for 45 minutes. The DO-7 antibody has been shown to bind to both abnormal "mutant" p53, and also to normal "wild type" p53. Bound antibody was visualised by immunoprecipitation with $\mathrm{DAB}$, and the cells lightly counterstained with haematoxylin. The percentage of p53 positive nuclei in each treatment group was determined by counting a minimum of five high power fields per well at each time point.

\section{Results}

Following all doses of irradiation, a significant reduction in HTF numbers was seen at all time points compared with untreated control cells (ANOVA with Bonferroni modification, $\mathrm{p}<0.001$ at all time points, Fig 1). Control HTFs showed exponential growth up to day 14 , after which the cells reached confluence and the growth rate reached a plateau. Following $250 \mathrm{cGy}$ of $\beta$ radiation there was a reduced but continued degree of cell proliferation over the time course studied, but after larger doses of radiation, the rate of fibroblast proliferation was significantly reduced at all time points. For all radiation doses studied, no significant recovery in fibroblast proliferation was seen over the duration of the experiment. Despite the profound antiproliferative effect, vital dye staining of the monolayers with trypan blue showed no significant difference in the percentage of dead cells between control and treated groups throughout the time course of the study, although for doses of radiation in excess of 750 cGy there was a small but significant reduction in cell numbers from the starting level by 28 days (Student's $t$ test $\mathrm{p}<0.01$ ).

During the period of exponential phase growth, numerous mitotic figures were visible in all the control fibroblast monolayers. In all irradiated cultures however, a marked reduction in mitotic figures was noted from day 1, with no mitoses visible from 24 hours following doses in excess of $750 \mathrm{cGy}$ of $\beta$ radiation. In situ BRdU labelling of untreated control cells showed that up to $70 \%$ of the cells were cycling in the first 24 hours, reducing to $51 \%$ at 24 hours. There was subsequently a gradual decline in the cycling index of the control cells as the population achieved confluence. In the irradiated cells the percentage of fibroblasts actively cycling was found to be significantly reduced following all doses studied, with a significant effect detectable from as soon as 4 hours after irradiation. Only $17 \%$ of HTFs were found to be cycling 4 hours after 1000 cGy, and this reduced to $5 \%$ by 24 hours and $1 \%$ by 48 hours (Fig 2). No significant recovery was seen in the cycling index over the entire 28 day period following doses of 1000 and $2000 \mathrm{cGy} \beta$ radiation.

Wild type p53 was undetectable by immunocytochemistry in normal untreated fibroblasts, whereas irradiated fibroblasts showed a marked increase in positive p53 staining by 24 


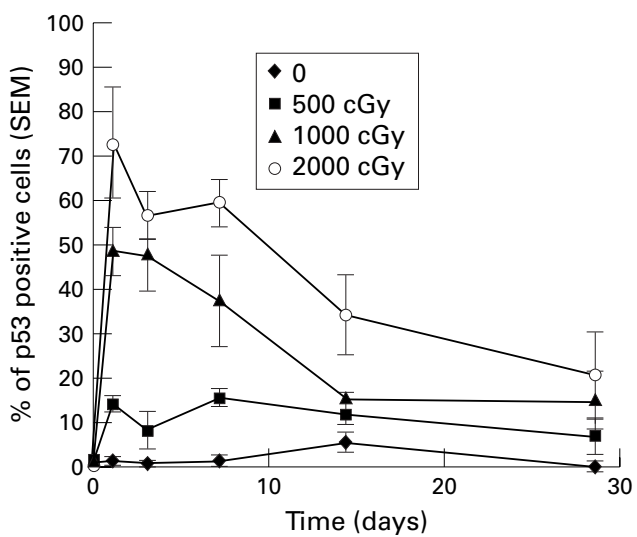

Figure 4 Effects of $\beta$ radiation on $p 53$ expression. Monolayers of exponentially growing fibroblasts were prepared and treated as before. At each time point triplicate wells from each treatment group and control groups were fixed in 50:50 acetonelethanol and then immunostained with Dako DO-7 anti-p53 antibody. Bound antibody was visualised using $D A B$ immunoprecipitation and the percentage of positively staining cells was determined for each treatment and plotted against time.

hours following treatment (Fig 3). All irradiated populations showed evidence of $\mathrm{p} 53$ positive cells, with greater levels following the higher doses of radiation. p53 levels remained detectable for up 14 days after irradiation, but then appeared to reduce to control levels (Fig $4)$.

\section{Discussion}

We have shown that treatment with single doses of $\beta$ radiation from a strontium-90 source can effectively inhibit the proliferation of human Tenon's capsule fibroblasts over a 28 day period in vitro. This finding both agrees with and extends the other published data into the effects of $\beta$ radiation on human and monkey fibroblasts. ${ }^{14}{ }^{15}$ After treatment with $250 \mathrm{cGy}$, the growth rate of the population of HTF was retarded but not stopped, and a gradual increase in cell numbers was seen over the time course of the experiment. For doses in excess of 750 cGy, however, no significant increase in cell numbers from initial levels was seen, demonstrating the powerful antiproliferative effect of this treatment. Furthermore, we have shown that the inhibition of HTF proliferation persists for at least 28 days in vitro, with no late recovery in cell proliferation occurring.

Radiation is known to induce rapid cell death in some cell lines, including lymphocytes and endothelial cells, and this rapid onset interphase death has recently been shown to be the result of radiation triggered apoptosis. ${ }^{21} 22$ We therefore sought to determine if the reduction in fibroblast numbers which we had observed was a consequence of a similar increase in the rate of death in the irradiated cells. The trypan blue cell viability studies demonstrated, however, that the vast majority of the fibroblasts remained viable over the duration of the experiment. Furthermore, we found only a small drop in HTF numbers below starting levels following the highest doses of radiation that we studied. These findings suggest that for the clinically relevant doses of radiation investigated, ocular fibroblasts do not undergo high levels of interphase (apoptotic) or postmitotic (necrotic) death, but appear to enter a period of prolonged growth arrest. This finding may be very relevant for the inhibition of scarring, as high levels of necrotic death could in themselves induce more local scarring to occur.

BRdU is a thymidine analogue taken up by proliferating cells as they pass through the $\mathrm{S}$ phase of the cell cycle. Following a pulsed exposure in BRdU labelled medium, demonstration of incorporated BRdU by immunocytochemistry allows the cycling index of a population of cells to be determined, thereby indicating the proportion of cells that have passed through $\mathrm{S}$ phase over the "pulsing period". ${ }^{23}$ Our BRdU studies confirmed that the fibroblasts were indeed becoming "growth arrested" following irradiation, demonstrating a marked reduction in cell cycling in all the irradiated HTF populations. The onset of this cell cycle delay appears to be rapid, with a significant reduction in the cycling index relative to control cells detectable by 4 hours after 500 cGy irradiation. Analysis of the later time points failed to show any recovery in BRdU uptake, again demonstrating that this period of "growth arrest" is prolonged, with no appreciable recovery.

p53 is a nuclear phosphoprotein which has been shown to be involved in the control of cell cycling, both at the G1/S and possibly the $\mathrm{G} 2 / \mathrm{M}$ transitions in the cell cycle. ${ }^{24-26}$ Normally p53 is undetectable by immunocytochemistry in its natural or "wild type" form, although increased levels of mutated p53 have been found in many tumours, where they are thought to be associated with abnormal cell cycle control mechanisms. ${ }^{27}{ }^{28}$ Wild type p53 expression has been found to be increased in many cell types following DNA damage caused by a variety of agents, where it is thought to exert a "policing" function preventing replication of damaged DNA. Interestingly, mutations in the p53 gene such as those seen in Li-Fraumeni syndrome have been associated with an increased risk of developing cancer, and there is also evidence to suggest that the increased tumour susceptibility seen in ataxiatelangiectasia is the result of an impaired p53 response. ${ }^{29}{ }^{30}$ In addition to its effects on cell cycling, there is also evidence to suggest that increased p53 levels may also trigger apoptosis in certain cell lines. ${ }^{31}$ In this paper we have demonstrated that single doses of $\beta$ radiation increase the expression of $\mathrm{p} 53$ in normal ocular fibroblasts, presumably in response to the DNA damage caused by the radiation. Kuerbitz et al have demonstrated that the presence of a G1 cell cycle delay after irradiation is associated with an increase in p53 expression, ${ }^{26}$ and El Deiry et al have further demonstrated that the increased nuclear p53 itself leads to an increase in p21 (WAF1/CIP1) levels, a protein which directly inhibits the cyclin dependent kinases required for continued progression through the cell cycle. ${ }^{32}$ It appears likely therefore that activation of the p53 "guardian of the genome" mechanism is indeed one of the 
cellular triggers for growth arrest following $\beta$ irradiation in ocular fibroblasts. ${ }^{33}$ Furthermore, although the inhibition of ocular fibroblast cycling as a result of radiation induced DNA damage raises concerns into the long term safety of such treatments, the clinical experience of adjunctive $\beta$ radiation treatment with trabeculectomy in children in our unit has shown it to be well tolerated and without serious side effects.

In conclusion, we have shown that single doses of $\beta$ radiation can effectively inhibit ocular fibroblast proliferation for up to 28 days in vitro. We have also demonstrated that this response is not a consequence of high levels of cell death, but rather because the fibroblasts undergo a rapid onset and sustained period of growth arrest following doses of radiation comparable with those used clinically. In addition, we have shown that $\beta$ radiation causes an increase in cellular p53 expression, and it is possible that this is one of the cellular triggers of growth arrest in these cells. Interestingly, although agents such as 5-fluorouracil and mitomycin $\mathrm{C}$ can also induce prolonged growth arrest in ocular fibroblasts, it has recently been shown that they also interfere with other fibroblast functions such as migration and cell mediated contraction. ${ }^{34}{ }^{35} \mathrm{We}$ are currently investigating the effects of $\beta$ radiation on other fibroblast wound healing functions in our laboratory to establish if a different response occurs after radiation, as this may explain the differing bleb morphology seen after irradiation or antimetabolite treatment. It is hoped that an increased understanding of the cellular biology of the effects of radiation will enhance the development and clinical application of this potentially useful adjuvant therapy for modulating the healing response following trabeculectomy.

This work was supported by the TFC Frost Trust, the International Glaucoma Association, and the Wellcome Trust.

1 The Fluorouracil Filtering Surgery Study Group. Five-year follow-up of the fluorouracil filtering surgery study. $A m \mathcal{F}$ Ophthalmol 1996;121:349-66.

2 Katz GJ, Higginbotham EJ, Lichter PR, et al. Mitomycin C versus 5-fluorouracil in high-risk glaucoma filtering surgery. Ophthalmology 1995:102:1263-9.

3 Skuta GL, Beeson CC, Higginbotham EJ, et al. Intraoperative mitomycin versus postoperative 5-fluorouracil in hightive mitomycin versus postoperative 5 -fluorouracil in highrisk glauco 44 .

4 Newhouse RP, Beyrer C. Hypotony as a late complication of trabeculectomy. Ann Ophthalmol 1982;July:685-6.

5 Jampel HD, Pasquale LR, Dibernardo C. Hypotony macuJampel HD, Pasquale LR, Dibernardo C. Hypotony macu-
lopathy following trabeculectomy with mitomycin C. Arch lopathy following trabeculector
Ophthalmol 1992;110:1049-50.

6 Franks WA, Hitchings RA. Complications of 5-fluorouracil after trabeculectomy. Eye 1991;5:385-9.

7 Higginbotham EJ, Stevens RK, Musch DC, et al. Blebrelated endophthalmitis after trabeculectomy with mitomycin. Ophthalmology 1996;103:650-6.

8 Cooper JS. Postoperative irradiation of pterygia: ten more years of experience. Radiology 1978;28:753-6.
9 Aswad MI, Baum J. Optimal time for postoperative irradiation of pterygia. Ophthalmology 1987;94:1450-1.

10 Cameron M. Beta irradiation as an adjunct to surgery in refractory glaucoma. Trans Aust Coll Ophthalmol 1970;2 53-60.

11 Cohen LB, Graham FT, Fry WE. Beta radiation as an adjunct to glaucoma surgery in the negro. Am $\mathcal{F}$ Ophthalmol 1959;47:54-61

12 Iliff CE. Surgical control of glaucoma in the negro. $A m \mathcal{F}$ Ophthalmol 1944;27:731-8.

13 Miller MH, Rice NSC. Trabeculectomy combined with beta irradiation for congenital glaucoma. Br f Ophthalmol 1991; 75:584-90.

14 Nevarez JA, Parrish RK, Heuer DK, et al. The effect of beta irradiation on monkey Tenon's capsule fibroblasts in tissue culture. Curr Eye Res 1987;6:719-23.

15 Khaw PT, Ward S, Grierson I, et al. Effect of beta radiation on proliferating human Tenon's capsule fibroblasts. $\mathrm{Br} f$ Ophthalmol 1991;75:580-3.

16 Di Leonardo A, Linke SP, Clarkin K, et al. DNA damage triggers a prolonged p53-dependent G1 arrest and long-term induction of Cip1 in normal human fibroblasts. Genes Dev 1994;8:2540-51.

17 Lane DP. p53 guardian of the genome. Nature 1992;358:1516.

18 Chen PL, Chen Y, Bookstein R, et al. Genetic mechanisms of tumour suppression by the human p53 gene. Science 1990;250:1576-80.

19 Joseph JP, Grierson I, Hitchings RA. Normal rabbit aqueous humour, fibronectin and fibroblast conditioned medium are chemoattractant to Tenon's capsule fibroblasts. Eye 1987;1:585-92.

20 Khaw PT, Ward S, Porter A, et al. The long-term effects of 5 -fluorouracil and sodium butyrate on human Tenon's fibroblasts. Invest Ophthalmol Vis Sci 1992;33:2043-52.

21 Tauchi H, Sawada S. Analysis of mitotic cell death caused by radiation in mouse leukaemia L $5178 \mathrm{Y}$ cells: apoptosis is the ultimate form of cell death following mitotic failure. Int f Radiat Biol 1994;65:449-55.

22 Fuks Z, Persaud RS, Alfieri A, et al. Basic fibroblast growth factor protects endothelial cells against radiation-induced programmed cell death in vitro and in vivo. Cancer Res 1994;2582-90.

23 Gratzner HG, Leif RC, Ingram DJ, et al. The use of antibody specific for bromodeoxyuridine for the immunofluorescent determination of DNA replication in single cells and chromosomes. Exp Cell Res 1975;95:88-94.

24 Stewart N, Hicks GG, Paraskevas F, et al. Evidence for a second cell cycle block at G2/M by p53. Oncogene 1995;10: 109-15.

25 Agarwal ML, Agarwal A, Taylor WR, et al. p53 controls both the $\mathrm{G} 2 / \mathrm{M}$ and the $\mathrm{G} 1$ cell cycle checkpoints and mediates reversible growth arrest in human fibroblasts. Proc Natl Acad Sci USA 1995;92:8493-7.

26 Kuerbitz SJ, Plunkett BS, Walsh WV, et al. Wild-type p53 is a cell type checkpoint determinant following irradiation. a cell type checkpoint determinant followin
Proc Natl Acad Sci USA 1992;89:7491-5.

27 Tobal K, Warren W, Cooper CS, et al. Increased expression and mutation of $\mathrm{p} 53$ in choroidal melanoma. Br $\mathcal{F}$ Cancer 1992;66:900-4.

28 Hooper ML. The role of the p53 and Rb-1 genes in cancer, development and apoptosis. F Cell Sci 1994;18:13-17.

29 Kastan MB, Zhan Q, El-Deiry W, et al. A mammalian cell cycle checkpoint pathway utilizing p53 and GADD45 is defective in ataxia-telangiectasia. Cell 1992;71:587-97.

30 Enoch T, Norbury C. Cellular responses to DNA damage: cell-cycle checkpoints, apoptosis and the roles of p53 and ATM. TIBS 1995;20:426-30.

31 Lowe SW, Schmitt EM, Smith SW, et al. p53 is required for radiation-induced apoptosis in mouse thymocytes. Nature 1993;362:847-9.

32 El- Deiry WS, Tokino T, Velculescu VE, et al. WAF1, a potential mediator of p53 tumor suppression. Cell 1993;75: $817-25$.

33 Dulic V, Kaufmann WK, Wilson SJ, et al. p53-dependent inhibition of cyclin- dependent kinase activities in human fibroblasts during radiation induced $\mathrm{G} 1$ arrest. Cell 1994;76:1013-23.

34 Occleston NL, Alexander RA, Mazure A, et al. Effects of single exposures to antiproliferative agents on ocular fibroblast mediated collagen contraction. Invest Ophthalmol Vis Sci 1994;35:3681-90.

35 Occleston NL, Daniels JT, Tarnuzzer RW, et al. Single exposures to antiproliferatives: long term effects on ocular fibroblast wound healing behaviour. Invest Ophthalmol Vis Sci 1997; (in press). 